\title{
El acierto en el tiro libre en baloncesto: cómo influye el minuto de partido, el estado del marcador y ser equipo local o visitante
}

\author{
The successful free throw in basketball: \\ how it influences the minute of the game, the \\ scoreboard and be home or visitor team
}

\section{$\mathrm{O}$ acerto no lançamento livre do basquete como influi o minuto do jogo, o estado do jogador e ser equipe local ou visitante}

\author{
Manuel G. Jiménez-Torres y Carlos J. López Gutiérrez
}

Universidad de Granada

\begin{abstract}
Resumen
El objetivo de este estudio ha sido analizar los tiros libres lanzados en una muestra de 56 partidos de baloncesto de categoría ACB. Se ha registrado el número de lanzamientos y el porcentaje de aciertos en cada minuto de juego. Además, también se ha tenido en cuenta la situación del marcador en el momento de lanzar y la condición de actuar como equipo local o visitante. No se han encontrado diferencias significativas entre los equipos locales y los visitantes, en relación a los tiros libres intentados. En cuanto a los porcentajes de aciertos, existe una mínima diferencia favorable a los equipos que juegan como locales. Se comentan estos resultados y se hacen propuestas prácticas de cara al entrenamiento del tiro libre.

Palabras clave

Tiro libre, baloncesto, porcentaje de aciertos, análisis de juego, ventaja de jugar como local, equipo local, equipo visitante.

Abstract

The aim of this study was to analyze the released free throws in a sample of 56 basketball games category ACB. There has been the number of pitches and the percentage of hits in each minute of play. In addition, it also took into account the marker location at the time of launch and the condition of acting as the home team or visitor team. No significant differences were found between local
\end{abstract}

teams and visitors teams in relation to the free throw attempts. In terms of percentages of correct answers, there is minimal difference in favor of the teams playing at home. We discuss these findings and make practical proposals for the training side of the free kick.

Key words

Free shot, basketball, percentage of successes, game analysis, advantage of playing at home, home team, visitor team.

\section{Resumo}

O objetivo desse estudo foi analisar os lançamentos livres em uma mostra de 56 jogos de basquete da categoria ACB. Foram registrados os números de lançamentos e a porcentagem de acertos em cada minuto do jogo. Ademais foi considerada a situação do jogador no momento de lançar e a condição de atuar como equipe local ou visitante. Náo foram encontradas diferenças significativas entre as equipes locais ou visitantes, em relação às tentativas de lançamentos livres. Em relaçáo às porcentagens de acertos, existe uma mínima diferença favorável para as equipes que jogam como locais. Comentamos esses resultados e fazemos propostas de práticas enfocando ao treinamento de lançamentos livres.

Palavras chave: Lançamento livre, basquete, porcentagem de acertos, analises de jogo, vantagem de jogar em casa, equipe local, equipe visitante.

\section{Introducción}

El tiro libre es uno de los aspectos del baloncesto más estudiados. En él, el jugador se enfrenta en solitario a la canasta, sin oposición del contrario y siempre desde la misma distancia. Esta circunstancia ha suscitado el interés de estudios sobre la incidencia de diversos factores tales como la motivación, la concentración, el control de la ansiedad, etc. (Ittenbach y Esters, 1995; Fierro, 2002; Gómez y Lorenzo, 2007; Ibánez et

Jiménez-Torres, Manuel G. (Autor de contacto)

Departamento de Personalidad, Evaluación y Tratamiento Psicológico en la Facultad de Psicología. Universidad de Granada. Campus de Cartuja s/n, 18071 Granada (Espańa). Tel. (+34) 655987381. Email: mjitor@ugr.es al., 2008; Lorenzo, Gómez, Ortega, Ibánez y Sampaio, 2010; Sampaio, Drinkwater y Leite, 2010). Además, en el entorno del baloncesto también se le otorga una gran importancia a los tiros libres y prueba de ello son los registros habituales con respecto al porcentaje de aciertos y las consiguientes intervenciones para su mejora. Los entrenadores, en numerosos encuentros de competición, achacan la derrota a los bajos porcentajes obtenidos.

Así pues, está justificado otorgar a esta faceta del juego la importancia e incidencia que tiene sobre el resultado final del encuentro, tanto a nivel positivo como negativo. Algunos estudios señalan que el número de puntos que se obtiene en 
los encuentros a través del tiro libre supone entre el 20-25\% del total que se anota en un partido (Hays y Krause, 1987; Cárdenas y Rojas, 1997; Lorenzo, Gómez y Sampaio, 2003; Sampaio, Fraga y Silva, 2004), creciendo este porcentaje al $35 \%$ en los últimos cinco minutos de partido y hasta el 69\% en el último minuto de juego o en finales igualados (Kozar, Vaughn, Lord y Whitfield, 1995; Ibánez, García, Feu, Parejo y Cañadas, 2009; Navarro, Lorenzo, Gómez y Sampaio, 2009). En esos momentos de los partidos podemos afirmar que más de la mitad de la producción de puntos en los minutos finales son conseguidos a través del tiro libre.

Además, diferentes trabajos afirman que los tiros libres determinan el resultado de más de la mitad de los partidos jugados en una temporada (Walker, 1985; Hays y Krause, 1987). Jenkins (1977) añade que el equipo que obtiene mayor porcentaje en el tiro libre gana en un $80 \%$ de las ocasiones. Por otro lado, Ibánez et al. (2009) hacen mención a que determinadas situaciones contextuales pueden cambiar los aspectos estadísticos que discriminan sobre ganadores o perdedores.

Esta relevancia del tiro libre, unido a la consideración de la necesidad de un mayor control sobre las variables que influyen en la eficacia del acierto, hace que este tipo de lanzamiento presente estudios desde múltiples perspectivas. Así, a nivel biomecánico y de control motor, diferentes artículos nos muestran los aspectos técnicos de idoneidad en el lanzamiento de tiro libre, a través del análisis de modelos tridimensionales (Okubo y Hubband, 2006; Keetch, Lee y Schmidt, 2008; Tran y Silverberg, 2008; Schneider y Williams, 2010). Otros estudios plantean orientaciones enfocadas al entrenamiento sobre aspectos a tener en cuenta en la realización de las actividades que más se pudieran acercar al contexto de competición (Cárdenas, 1998; Getz y Rainey, 2001; Ortega, Cárdenas, Puigcerver y Méndez, 2005; Foster y Weigand, 2006; Lonsdale y Tam, 2008; Arias, Argudo y Alonso, 2012), o teniendo en cuenta los factores de tipo psicológico que se ven implicados en la ejecución de este patrón motor, como la atención, la concentración, el control visual, la ansiedad o los niveles de estrés (Rivares, 1996; Whitehead, Butz; Kozar y Vaughn, 1996; Dandy, Brewer y Tottman, 2001; Harle y Vickers, 2001; Larumbe, 2001; Oliveira, Oudejans y Beek, 2008; Mesagno, Marchant y Morris, 2009; Gooding y Gardner, 2009; Otten, 2009; Wilson, Vine y Wood, 2009; Krendl, Gainsburg y Ambady, 2012).

En definitiva, los investigadores han tratado de constatar cómo algunos factores pueden incidir en la eficacia del tiro libre, tanto factores externos (ej. la importancia del partido) como factores internos, personales (motivación, presión, etc.).

El tiro libre se considera un lanzamiento hasta cierto punto sencillo, sin embargo conlleva una gran carga de tipo psicológico que, en situaciones de competición, lo transforma en una tarea complicada al verse alterado por factores de estrés y fatiga (Sampaio y Janeira, 2003). Con relación a la fati- ga, diferentes estudios concluyen que, aunque ésta no tenga efectos directos sobre el acierto, puede actuar sobre variables psicológicas como la atención o la concentración que son de suma importancia para una correcta ejecución (Montgomery et al., 2008; Ibańez et al., 2009; Uygur, Gottepe, Karabörk y Korkusuz, 2010).

Los estudios relacionados con los aspectos psicológicos tratan de acercar la situación del tiro libre en el entrenamiento a las condiciones que se presentan en competición en cuanto a la ansiedad o el estrés, o bien de detectar qué elementos deben considerarse desde el punto de vista de la atención y la concentración que pudieran derivar en la mejora del rendimiento en la ejecución. Los factores que causan estrés son los que pueden afectar más a la eficacia del tiro libre.

Otro de los aspectos más importantes en el análisis de las estadísticas, surge en el estudio de las victorias y las derrotas de los equipos en función de la situación en la que juegan como local o visitante. Esta área de investigación se denomina home advantage effect. Este aspecto supone un fenómeno a tener en cuenta como factor que condiciona los partidos de baloncesto (Balmer, Nevill y Williams, 2001; Bray y Widmeyer, 2000; Courneya y Carron, 1992; Silva y Andrew, 1987). En baloncesto masculino el porcentaje de victorias por el hecho de jugar en casa se sitúa cerca del $64 \%$, mientras que en baloncesto femenino se sitúa en torno al 70\% (Courneya y Carron, 1992).

Más recientemente los estudios de Štrumbelj, Vračar, Robnik-Šikonja, Dežman y Erčulj (2011) sitúan este porcentaje, en lo que a la liga eslovena se refiere en un 59\% en categoría masculina, coincidiendo con los estudios en Euroliga de Gómez y Pollard (2011) que establece el 58\%. Otros estudios destacan que los registros que mejor discriminan a los equipos que juegan como locales son unos mejores porcentajes de acierto en tiros de campo y tiros libres (Pickens, 1994; Moore y Brylinsky, 1995).

Los estudios que destacan la ventaja de jugar en casa argumentan como factores favorables el ahorro del desgaste del desplazamiento, el conocimiento de la cancha de juego, la presión percibida por los árbitros, la familiaridad con el estadio, el público y determinadas variables psicológicas (ansiedad, estados de ánimo) y conductuales (Sánchez et al., 2001; Smith, 2005). También argumentan estos estudios que el hecho de jugar fuera suele influir psicológicamente en el equipo, afectando a su disposición motivacional y debilitando algunas de las variables psicológicas más importantes relacionadas con el rendimiento como la concentración, el control del estrés y los estados anímicos.

También ha sido objeto de estudio las diferencias en el marcador a la hora de lanzar un tiro libre. Ahart (1973) encontró que el porcentaje de tiros libres se optimizaba cuando las diferencias en el marcador oscilaban entre 5 y 8 puntos, es decir, cuando puede considerarse que hay una presión inter- 
media por la necesidad de anotar el tiro. La efectividad era más baja cuando el marcador señalaba una diferencia más pequeña de 5 puntos, en cuyo caso la presión por anotar es mayor, y cuando la diferencia mayor de 8 , de modo que la presión en esta situación es muy baja. En consonancia con los datos anteriores está el estudio de Lafuente (2005). Otros estudios discrepan de los anteriores. Algunos de ellos afirman que los porcentajes de aciertos más altos se dan cuando el marcador está más igualado, entre 1 y 3 puntos de diferencia. Estos resultados pueden ser interpretados desde la influencia de la motivación y la concentración en la eficacia deportiva (Buceta, 1992; Labrador, Crespo, Buceta y González; 1995).

Otro aspecto estudiado es el número de lanzamientos y porcentaje de aciertos en el tiro libre según el minuto de partido que se esté jugando y la repercusión de todas estas circunstancias en la victoria final. Han sido varios los estudios que han seńalado la importancia del tiempo de partido para la eficacia del enceste del tiro libre en el baloncesto. Kozar, Vaughn, Whitfield y Lord (1994) encontraron, en un análisis de casi 500 partidos, que en los últimos 5 minutos se produce una proporción de lanzamientos de tiros libres más alta que en los 35 minutos precedentes. Labrador et al. (1995) señalan cierta tendencia a un empeoramiento en la efectividad del tiro libre a medida que avanza el partido. Igualmente encuentran mejores porcentajes en el tiro en los 5 primeros minutos de cada parte, con respecto a los 5 últimos minutos. En el mismo estudio, teniendo en cuenta el marcador del partido, se encuentra una efectividad muy alta en el tiro libre en situaciones de remontada.

En cuanto a la repercusión del tiro libre en la victoria final, Cárdenas y Rojas (1997) señalan que los equipos ganadores consiguen realizar un mayor número de lanzamientos en relación a los equipos que pierden e igualmente consiguen convertir un mayor número de lanzamientos. Lo verdaderamente determinante a estos niveles no es el porcentaje de eficacia, que suele ser muy similar, sino el número de lanzamientos que el equipo llega a ejecutar. Según estos investigadores, el porcentaje de puntos que se obtuvieron a través de tiros libres con respecto al cómputo final de puntos obtenido por los equipos ganadores fue del $21,85 \%$ frente a un $21,01 \%$ de los equipos que perdieron.

Teniendo en cuenta todas las consideraciones anteriores, en el presente estudio pretendemos analizar la eficacia en el lanzamiento del tiro libre en función de las diferencias en el marcador así como el tiempo de juego dentro de competiciones donde cada partido es de máxima relevancia, como son los play offs en la categoría ACB.

Específicamente nos hemos propuesto los siguientes objetivos:

1.- Analizar el número de tiros libres efectuados y el porcentaje de aciertos en función del minuto de juego en que se ejecuta el lanzamiento.
2.- Determinar si existen diferencias en el porcentaje de aciertos en tiros libres en función de la situación del marcador en el momento de lanzar.

3.- Estudiar la influencia de actuar como local o visitante en el número de tiros libres efectuados y el porcentaje de aciertos.

4.- Comprobar si la relevancia del partido para resolver la eliminatoria guarda relación con el acierto en el tiro libre y con la victoria final local o visitante.

\section{Método}

\section{Diseño}

Se trata de un estudio ex post facto bibliométrico, según la clasificación de Montero y León (2002). En él se analizan relaciones entre variables en una situación ya pasada a la que se accede a través de diversas fuentes documentales (en nuestro caso, las estadísticas oficiales de la Asociación de Clubes de Baloncesto, ACB).

\section{Muestra}

Se ha tomado como muestra los 56 partidos de baloncesto ACB correspondientes a los playoffs de los ańos 2008, 2009 y 2010 (ver Apéndice). El 30,1\% de partidos corresponde a la temporada $2007-2008$, el 35,8\% a la $2008-2009$ y el $34,1 \%$ a la 2009-2010. El 51,5\% de partidos corresponde al cruce de cuartos de final, el $30,6 \%$ a semifinal y el $17,9 \%$ a final.

\section{Instrumentos}

Se han utilizado para el presente estudio la base de datos de jugadas del partido de las estadísticas oficiales de la Asociación de Clubes de Baloncesto (ACB), así como una hoja de registro confeccionada ad hoc para registrar los tiros libres lanzados en cada minuto de juego.

\section{Procedimiento}

Para cada encuentro se realizó una hoja de registro donde se tomaban en cuenta las siguientes variables: competición y temporada; eliminatoria, equipos implicados, orden del partido; minuto de juego; número de lanzamientos de tiro libre, marcador y parcial entre los equipos en el momento del tiro libre, número de tiros anotados, porcentaje, y resultado final. En la hoja de registro quedaron reflejados todos los elementos estadísticos que luego se volcaron sobre una matriz en el programa SPSS.

\section{Resultados}


El tratamiento estadístico de los datos se ha realizado con el programa SPSS 15.0 para Windows. Previamente se ha llevado a cabo análisis preliminares y exploratorios para conocer las características de los datos. Habiendo obtenido que se cumple el criterio de normalidad (prueba de KolmogorovSmirnov p > ,05), hemos optado por utilizar pruebas paramétricas en los análisis estadísticos. El nivel de significación para todos los análisis se fijó en $p<, 05$. Se han llevado a cabo análisis descriptivos y análisis comparativos mediante ANOVA y $t$ de Student.

\section{Número de lanzamientos y porcentajes de aciertos}

En la Tabla 1 se muestran los tiros lanzados en los diferentes intervalos de tiempo analizados tanto por el equipo local como visitante. También se refleja el porcentaje de aciertos de ambos equipos en ese mismo intervalo de tiempo. En los 56 partidos analizados, los equipos locales lanzaron un total de 1155 tiros libres mientras que el número de lanzamientos de los equipos visitantes fue de 1064. En los intervalos de 5 minutos se observa un patrón de línea quebrada de tal manera que en las primeras mitades de los cuartos suelen producirse menor número de lanzamientos que en las segundas mitades de los mismos.
Además, a medida que avanza el partido en cuartos y tiempos también se van incrementando el número de lanzamientos. Así, mientras que en el primer tiempo los equipos que actuaron de locales realizaron un total de 456 lanzamientos de tiros libres y los que actuaron como visitantes lanzaron 433 tiros libres, en el segundo tiempo las cifras anteriores se incrementaron a 684 y 604 respectivamente para los equipos locales y visitantes.

Por otro lado, en cuanto al porcentaje medio de aciertos, no se observan diferencias apreciables entre los primeros y segundos tiempos de los partidos, ni en el equipo local ni en el visitante. El porcentaje medio total es 76,46 y 77,69 para los primeros y segundos tiempos respectivamente. No obstante, locales y visitantes siguen una tendencia contraria: el local suele incrementar ligeramente su porcentaje de aciertos en el segundo tiempo con respecto al primero $(78,50$ frente a 76,00$)$, mientras que en el visitante suele descender ligeramente $(76,88$ frente a 76,92$)$.

Otro dato llamativo es que, cuando se ha producido prórroga, los locales suelen bajar el porcentaje de aciertos en ese periodo de prórroga con respecto al segundo tiempo $(69,50$ frente a 78,50), mientras que los visitantes suelen mejorar sus aciertos en dicho periodo en comparación a sus registros del segundo tiempo (85,00 frente a 76,88).

Tabla 1. Tiros libres lanzados y porcentajes de aciertos en cada periodo de tiempo

\begin{tabular}{|c|c|c|c|c|c|c|}
\hline \multirow{2}{*}{$\begin{array}{l}\text { CADA } \\
\text { CINCO } \\
\text { MINUTOS }\end{array}$} & \multicolumn{3}{|c|}{ No de Lanzamientos } & \multicolumn{3}{|c|}{ Porcentaje medio de aciertos } \\
\hline & Local & Visitante & Total & Local & Visitante & Total \\
\hline Minuto $1-5$ & 72 & 60 & 132 & 80,62 & 80,48 & 80,55 \\
\hline Minuto 6-10 & 149 & 110 & 259 & 79,58 & 71,36 & 75,47 \\
\hline Minuto $11-15$ & 77 & 95 & 172 & 69,77 & 77,50 & 73,63 \\
\hline Minuto $16-20$ & 158 & 168 & 326 & 73,59 & 78.90 & 76,24 \\
\hline Minuto 21-25 & 98 & 91 & 189 & 85,42 & 76,98 & 81,20 \\
\hline Minuto 26-30 & 179 & 183 & 362 & 77,40 & 79,14 & 78,27 \\
\hline Minuto 31-35 & 125 & 89 & 214 & 68,86 & 70,51 & 69,68 \\
\hline Minuto 36-40 & 282 & 241 & 523 & 81,93 & 79,87 & 80,90 \\
\hline Minuto $41-45$ & 15 & 27 & 42 & 69,50 & 85,00 & 77,25 \\
\hline \multicolumn{7}{|l|}{ CUARTOS } \\
\hline Primer Cuarto & 221 & 170 & 391 & 79,95 & 74,91 & 77,43 \\
\hline Segundo Cuarto & 235 & 263 & 498 & 72,15 & 78,15 & 75,15 \\
\hline Tercer Cuarto & 277 & 274 & 551 & 80,25 & 76,66 & 78,45 \\
\hline Cuarto Cuarto & 407 & 330 & 737 & 77,22 & 77,03 & 77,12 \\
\hline Prórroga & 15 & 27 & 42 & 69,5 & 85,00 & 77,25 \\
\hline \multicolumn{7}{|l|}{ TIEMPOS } \\
\hline Primer Tiempo & 456 & 433 & 889 & 76 & 76,92 & 76,46 \\
\hline Segundo tiempo & 684 & 604 & 1288 & 78,50 & 76,88 & 77,69 \\
\hline
\end{tabular}




\begin{tabular}{lcccccc}
\hline CADA & \multicolumn{3}{c}{ No de Lanzamientos } & \multicolumn{3}{c}{ Porcentaje medio de aciertos } \\
\cline { 2 - 7 } $\begin{array}{l}\text { CINCO } \\
\text { MINUTOS }\end{array}$ & Local & Visitante & Total & Local & Visitante & Total \\
\hline Prórroga & 15 & 27 & 42 & 69,5 & 85,00 & 77,25 \\
\hline
\end{tabular}

$\mathrm{N}=56$ partidos (4 partidos con prórroga).

Análisis comparativos de los porcentajes de aciertos en diferentes tiempos

En la tabla 2 se exponen los resultados de los diferentes análisis comparativos efectuados. Las comparaciones se han llevado a cabo para cada una de las diferentes fracciones de tiempo registradas, y separadamente para los equipos locales y visitantes. Como puede apreciarse en dicha tabla, ninguna de las comparaciones efectuadas ha resultado significativa (todos los p-valores resultaron $>0,05$ ). Así pues, tanto para los equipos locales como para los visitantes, no podemos afirmar que un porcentaje de aciertos en un determinado intervalo de tiempo sea superior o inferior al que se haya producido en otro intervalo.

A pesar de lo anterior, como dato curioso podemos observar cómo los equipos locales alcanzan sus máximos porcentajes de aciertos en los segundos tiempos de los partidos $(78,50)$, en los terceros cuartos $(80,25)$ y concretamente entre los minutos 21 y 25 de los partidos $(85,42)$. En cambio, los equipos visitantes alcanzan sus máximos porcentajes de aciertos en los primeros tiempos de los partidos $(76,92)$, en los segundos cuartos $(78,15)$, aunque concretamente ha sido entre los minutos 1 y 5 de los partidos cuando alcanzaron sus máximos porcentajes de aciertos $(80,48)$.

En los cuatro partidos en que se ha producido prórroga, los equipos locales obtuvieron una media de aciertos desde el tiro libre del 69,50\%, mientras que los equipos visitantes alcanzaron el 85,00\%.

Las medias de aciertos desde el tiro libre obtenidas al final del partido por los equipos locales y visitantes fueron respectivamente del $77,34 \%$ y $77,05 \%$.

Al comparar las medias de aciertos en tiros libres obtenidos en los primeros cinco minutos de los partidos con las medias alcanzadas en los cinco últimos minutos, tampoco se han encontrado diferencias significativas ni en los equipos locales $(80,63$ frente a 81,93$)$ ni en los visitantes $(80,49$ frente a 79,97).

Tabla 2. Porcentajes de aciertos en tiro libre en cada periodo de tiempo

\begin{tabular}{|c|c|c|c|c|c|c|}
\hline & TIEMPOS & $\mathrm{N}$ & Media & $\begin{array}{l}\text { Desv. } \\
\text { Típica }\end{array}$ & $F$ & $\begin{array}{c}\text { Sig. } \\
p\end{array}$ \\
\hline \multirow{9}{*}{$\begin{array}{l}\text { Porcentajes } \\
\text { de aciertos } \\
\text { (Local) }\end{array}$} & Minuto 1-5 & 56 & 80,63 & 33,25 & \multirow{9}{*}{1,92} & \multirow{9}{*}{, 06} \\
\hline & Minuto 6-10 & 56 & 79,58 & 30,85 & & \\
\hline & Minuto $11-15$ & 56 & 69,77 & 39,55 & & \\
\hline & Minuto $16-20$ & 56 & 73,59 & 32,88 & & \\
\hline & Minuto 21-25 & 56 & 85,42 & 23,23 & & \\
\hline & Minuto 26-30 & 56 & 77,11 & 29,22 & & \\
\hline & Minuto 31-35 & 56 & 68,86 & 35,16 & & \\
\hline & Minuto 36-40 & 56 & 81,93 & 25,48 & & \\
\hline & Minuto 41-45 & 4 & 69,50 & 40,02 & & \\
\hline
\end{tabular}




\begin{tabular}{|c|c|c|c|c|c|c|}
\hline & TIEMPOS & $\mathrm{N}$ & Media & $\begin{array}{l}\text { Desv. } \\
\text { Típica }\end{array}$ & $F$ & $\begin{array}{l}\text { Sig } \\
p\end{array}$ \\
\hline \multirow{9}{*}{$\begin{array}{l}\text { Porcentajes } \\
\text { de aciertos } \\
\text { (Visitante) }\end{array}$} & Minuto 1-5 & 56 & 80,49 & 32.20 & \multirow{9}{*}{0,95} & \multirow{9}{*}{, 47} \\
\hline & Minuto 6-10 & 56 & 71,36 & 32,42 & & \\
\hline & Minuto 11-15 & 56 & 77,50 & 30,41 & & \\
\hline & Minuto $16-20$ & 56 & 78,90 & 27,52 & & \\
\hline & Minuto 21-25 & 56 & 71,98 & 33,10 & & \\
\hline & Minuto 26-30 & 56 & 79,14 & 31,13 & & \\
\hline & Minuto 31-35 & 56 & 70,51 & 36,67 & & \\
\hline & Minuto 36-40 & 56 & 79,87 & 28,30 & & \\
\hline & Minuto 41-45 & 4 & 85,00 & 21,41 & & \\
\hline \multirow{6}{*}{$\begin{array}{l}\% \text { aciertos } \\
\text { (Local) }\end{array}$} & Primer cuarto & 56 & 79,95 & 31,59 & \multirow{6}{*}{1,37} & \multirow{6}{*}{, 24} \\
\hline & Segundo cuarto & 56 & 72,15 & 35,42 & & \\
\hline & Tercer cuarto & 56 & 80,25 & 27,32 & & \\
\hline & Cuarto cuarto & 56 & 77,22 & 29,90 & & \\
\hline & Prórroga & 4 & 69,50 & 40,2 & & \\
\hline & Total & 56 & 77,34 & 31,10 & & \\
\hline \multirow{6}{*}{$\begin{array}{l}\% \text { aciertos } \\
\text { (Visitante) }\end{array}$} & Primer cuarto & 56 & 74,91 & 32,47 & \multirow{6}{*}{0,34} & \multirow{6}{*}{, 85} \\
\hline & Segundo cuarto & 56 & 78,35 & 28,58 & & \\
\hline & Tercer cuarto & 56 & 76,66 & 31,88 & & \\
\hline & Cuarto cuarto & 56 & 77,04 & 31,24 & & \\
\hline & Prórroga & 4 & 85,00 & 21,42 & & \\
\hline & Total & 56 & 77,05 & 30,74 & & \\
\hline \multirow{3}{*}{$\begin{array}{l}\% \text { aciertos } \\
\text { (Local) }\end{array}$} & & & & & $t$ & $p$ \\
\hline & Primer tiempo & 56 & 76,00 & 33,74 & \multirow{2}{*}{$-0,91$} & \multirow{2}{*}{, 36} \\
\hline & Segund.tiempo & 56 & 78,50 & 28,84 & & \\
\hline \multirow{2}{*}{$\begin{array}{l}\% \text { aciertos } \\
\text { (Visitante) }\end{array}$} & Primer tiempo & 56 & 76,92 & 30,23 & \multirow{2}{*}{0,02} & \multirow{2}{*}{,98 } \\
\hline & Segund.tiempo & 56 & 76,86 & 31,48 & & \\
\hline \multirow{2}{*}{$\begin{array}{l}\% \text { aciertos } \\
\text { (Local) }\end{array}$} & Minuto 1-5 & 56 & 80,63 & 33,25 & \multirow{2}{*}{$-0,25$} & \multirow{2}{*}{, 80} \\
\hline & Minuto 36-40 & 56 & 81,93 & 25,48 & & \\
\hline \multirow{2}{*}{$\begin{array}{l}\% \text { aciertos } \\
\text { (Visitante) }\end{array}$} & Minuto 1-5 & 56 & 80,49 & 32,20 & \multirow{2}{*}{0,11} & \multirow{2}{*}{ 91 } \\
\hline & Minuto 36-40 & 56 & 79,97 & 28,30 & & \\
\hline
\end{tabular}

$p>, 05$. (Diferencias no significativas).

\section{Porcentaje de aciertos y situación del marcador}

Como se muestra en la tabla 3, los equipos locales lanzaron un total de 1155 tiros libres. El mayor número de ellos (296) lo hicieron cuando iban ganando entre 1 y 5 puntos. $56 \%$ de las veces lograron el 100\% de aciertos (648 de 1155); $25,3 \%$ de las veces lograron el $50 \%$ de aciertos (292 de 1155 ) y $8,1 \%$ de las veces se quedaron en el $0 \%$ de aciertos (94 de 1155). El menor porcentaje de veces que los equipos locales lograron el $100 \%$ de aciertos se produjo cuando iban perdiendo entre 6 y 10 puntos (40 veces de 89 posibles), mientras que el mayor porcentaje de veces que lograron el $100 \%$ de aciertos se produjo cuando lanzaron tiros libres con el marcador perdiendo por más de 10 puntos (50 veces de 85 posibles). El menor porcentaje de veces que los equipos locales fallaron todos los tiros libres se produjo cuando iban perdiendo entre 6 y 10 puntos (4 veces de 89 posibles), mientras que el mayor porcentaje de veces que fallaron todos los tiros libres se produjo cuando lanzaron con el marcador empatado (10 veces de 86 posibles).

Los equipos locales lograron el $100 \%$ de aciertos, con el marcador a favor el $59,6 \%$ de las veces, con el marcador en contra el $32,7 \%$ de las veces, y con empate en el marcador 
$7,7 \%$ de las veces. Por el contrario, estos equipos fallaron todos sus lanzamientos desde el tiro libre, con el marcador a favor $59,6 \%$ de las veces, con el marcador en contra 29,8\% de las veces y con empate en el marcador $10,6 \%$ de las veces.

Tabla 3. Porcentaje de aciertos y marcador al lanzar (equipo local)

\begin{tabular}{|c|c|c|c|c|c|c|c|c|c|}
\hline \multirow[b]{2}{*}{$\begin{array}{l}\text { Porcentajes } \\
\text { Aciertos }\end{array}$} & \multicolumn{9}{|c|}{ Marcador local } \\
\hline & & $\begin{array}{l}\text { Pierde } \\
+10\end{array}$ & $\begin{array}{c}\text { Pierde } \\
6-10\end{array}$ & $\begin{array}{c}\text { Pierde } \\
1-5\end{array}$ & Empate & $\begin{array}{c}\text { Gana } \\
1-5\end{array}$ & $\begin{array}{c}\text { Gana } \\
6-10\end{array}$ & $\begin{array}{c}\text { Gana } \\
+10\end{array}$ & Total \\
\hline \multirow{7}{*}{$\begin{array}{l}\text { Equipo } \\
\text { Local }\end{array}$} & 0 & 8 & 4 & 16 & 10 & 32 & 12 & 12 & 94 \\
\hline & $1-49$ & 1 & 4 & 2 & 1 & 1 & 5 & 4 & 18 \\
\hline & 50 & 26 & 26 & 62 & 12 & 66 & 52 & 48 & 292 \\
\hline & $51-74$ & 0 & 5 & 8 & 7 & 11 & 7 & 1 & 39 \\
\hline & 75 & 0 & 10 & 10 & 6 & 10 & 8 & 6 & 50 \\
\hline & $76-99$ & 0 & 0 & 0 & 0 & 8 & 6 & 0 & 14 \\
\hline & 100 & 50 & 40 & 122 & 50 & 168 & 126 & 92 & 648 \\
\hline Total & & 85 & 89 & 220 & 86 & 296 & 216 & 163 & 1155 \\
\hline
\end{tabular}

En la Tabla 4 se muestran los datos referentes a los equipos visitantes. Estos equipos lanzaron un total de 1064 tiros libres. El mayor número de ellos (276) lo hicieron cuando iban perdiendo entre 1 y 5 puntos. 55,8\% de las veces lograron el $100 \%$ de aciertos (594 de 1064); 26,7\% de las veces lograron el $50 \%$ de aciertos (284 de 1064 ) y $7 \%$ de las veces se quedaron en el $0 \%$ de aciertos (75 de 1064). El menor porcentaje de veces que los equipos visitantes lograron el $100 \%$ de aciertos se produjo cuando iban perdiendo entre 6 y 10 puntos (106 veces de 211 posibles), mientras que el mayor porcentaje de veces que lograron el $100 \%$ de aciertos se produjo cuando lanzaron tiros libres con el marcador en contra entre 1 y 5 puntos (170 veces de 276 posibles). El menor porcentaje de veces que los equipos visitantes fallaron todos los tiros libres se produjo cuando el marcador iba empatado (3 veces de 58 posibles), mientras que el mayor porcentaje de veces que fallaron todos los tiros libres se produjo cuando lanzaron con el marcador ganando por más de 10 puntos (5 veces de 42 posibles). Los equipos visitantes lograron el $100 \%$ de aciertos, con el marcador a favor el $27,6 \%$ de las veces, con el marcador en contra el $67,3 \%$ de las veces, y con empate en el marcador el $5,1 \%$ de las veces. Por el contrario, estos equipos fallaron todos sus lanzamientos desde el tiro libre, con el marcador a favor $33,3 \%$ de las veces, con el marcador en contra $62,6 \%$ de las veces y con empate en el marcador $4,1 \%$ de las veces.

Tabla 4. Porcentaje de aciertos y marcador al lanzar (equipo visitante)

\begin{tabular}{|c|c|c|c|c|c|c|c|c|c|}
\hline \multirow[b]{2}{*}{$\begin{array}{l}\text { Porcentajes } \\
\text { Aciertos }\end{array}$} & \multicolumn{9}{|c|}{ Marcador visitante } \\
\hline & & $\begin{array}{l}\text { Pierde } \\
+10\end{array}$ & $\begin{array}{c}\text { Pierde } \\
6-10\end{array}$ & $\begin{array}{c}\text { Pierde } \\
1-5\end{array}$ & Empate & $\begin{array}{c}\text { Gana } \\
1-5\end{array}$ & $\begin{array}{c}\text { Gana } \\
6-10\end{array}$ & $\begin{array}{c}\text { Gana } \\
+10\end{array}$ & Total \\
\hline \multirow{7}{*}{$\begin{array}{l}\text { Equipo } \\
\text { Visitante }\end{array}$} & 0 & 15 & 11 & 21 & 3 & 15 & 5 & 5 & 75 \\
\hline & $1-49$ & 3 & 3 & 5 & 0 & 3 & 4 & 0 & 18 \\
\hline & 50 & 56 & 80 & 62 & 16 & 42 & 24 & 4 & 284 \\
\hline & $51-74$ & 13 & 4 & 5 & 4 & 6 & 1 & 1 & 34 \\
\hline & 75 & 7 & 7 & 13 & 5 & 7 & 7 & 7 & 53 \\
\hline & $76-99$ & 0 & 0 & 0 & 0 & 0 & 6 & 0 & 6 \\
\hline & 100 & 124 & 106 & 170 & 30 & 91 & 48 & 25 & 594 \\
\hline Total & & 218 & 211 & 276 & 58 & 164 & 95 & 42 & 1064 \\
\hline
\end{tabular}

Como se muestra en el ANOVA de la Tabla 5, el estado del marcador no ha influido significativamente en el porcentaje de aciertos del equipo local $(F=0,21 ; \mathrm{p}>0,05)$ ni del equipo visitante $(F=0,37 ; \mathrm{p}>0,05)$. No obstante, se puede apreciar que el marcador favorable entre 6 y 10 puntos es la situación que se asocia con una media más elevada de aciertos en los 
equipos locales $(80,00 \%)$, mientras que ir ganando de más de de aciertos más alta en los equipos visitantes (82,35\%). 10 puntos es la situación del marcador asociada a una media

Tabla 5. Porcentajes de aciertos en tiro libre y marcador al lanzar

\begin{tabular}{lccccc}
\hline & Marcador & Media & Desv. Típica & $F$ & Sig. $p$ \\
\hline & Perdiendo +10 & 76,92 & 32,13 & & \\
& Perdiendo 6-10 & 76,00 & 27,08 & & \\
Porcentajes & Perdiendo 1-5 & 77,02 & 30,57 & & \\
de aciertos & Empate & 79,30 & 33,36 & \multirow{2}{*}{, 21 } &, 97 \\
(Equipo & Ganando 1-5 & 75,96 & 33,56 & & \\
Local) & Ganando 6-10 & 80,00 & 28,91 & & \\
& Ganando +10 & 76,75 & 31,02 & & \\
& Total & 77,34 & 31,10 & & \\
& Perdiendo +10 & 77,28 & 30,69 & & \\
Porcentajes & Perdiendo 6-10 & 74,00 & 29,60 & & \\
de aciertos & Perdiendo 1-5 & 78,76 & 31,29 & & \\
(Equipo & Empate & 78,83 & 28,33 & \multirow{2}{*}{0,37} & \\
Visitante) & Ganando 1-5 & 75,43 & 32,97 & & \\
& Ganando 6-10 & 78,14 & 28,81 & & \\
& Ganando +10 & 82,35 & 33,96 & & \\
\hline
\end{tabular}

$p>, 05$. (Diferencias no significativas).

\section{Puntos totales finales y puntos en tiros libres}

En cuanto a la comparación de puntos conseguidos por cada equipo, como puede observarse en la Tabla 6 , los equipos locales consiguen al final del partido una media de 79,32 puntos y de ellos 16,07 han sido obtenidos desde el punto de tiros libres (el 20\% aproximadamente).

Por su parte, los equipos visitantes consiguen una media de 74,00 puntos al final del partido, y de ellos 14,68 han sido obtenidos desde el punto de tiros libres (el 19,8\%). La media de tiros libres lanzados por el equipo local y visitante ha sido respectivamente 20,84 y 19,07 .

Tabla 6. Puntos al final del partido, tiros libres lanzados, tiros libres encestados y porcentaje de aciertos en tiros libres (N=56 partidos).

\begin{tabular}{lcccccc}
\hline & Equipo & N & Mínimo & Máximo & Media & Desv. típ. \\
\hline \multirow{2}{*}{ Puntos finales de partido } & Local & 56 & 47 & 102 & 79,32 & 11,17 \\
& Visitante & 56 & 53 & 97 & 74,00 & 11,09 \\
\multirow{2}{*}{ Tiros libres lanzados } & Local & 56 & 7 & 42 & 20,84 & 7,71 \\
& Visitante & 56 & 7 & 41 & 19,07 & 7,88 \\
\multirow{2}{*}{ Tiros libres encestados } & Local & 56 & 4 & 32 & 16,07 & 6,38 \\
\multirow{2}{*}{$\begin{array}{l}\text { Porcentajes de aciertos } \\
\text { en tiros libres }\end{array}$} & Visitante & 56 & 5 & 36 & 14,68 & 6,59 \\
\hline
\end{tabular}

En la Tabla 7 se muestran los puntos al final del partido, tiros libres lanzados, tiros libres encestados y porcentaje de aciertos en tiros libres en función del equipo ganador (local o visitante). La victoria local ha ocurrido en 38 de los 56 partidos analizados (el 68\% de las veces aproximadamente). En estas situaciones de victoria local, el equipo local lanzó una media de 21,21 tiros libres y acertó 16,58 (el 76,26\%) mientras que el equipo visitante lanzó 18,58 tiros libres de los cuales acertó 
14 (el 75,29\%). La victoria visitante ha ocurrido en 18 de los 56 partidos analizados (el 32\% de las veces aproximadamente). En estas situaciones de victoria visitante, el equipo local lanzó una media de 20,06 tiros libres y acertó 15 (el 75,78\%) mientras que el equipo visitante lanzó 20,11 tiros libres de los cuales acertó 16,11 (el 79,44\%).
Por lo que respecta a los puntos finales conseguidos, el equipo local ha conseguido una media de 82,55 puntos cuando ha conseguido la victoria y 72,50 cuando ha sido derrotado; por su parte, el equipo visitante ha conseguido una media de 80,22 puntos en caso de victoria y de 71,05 cuando ha perdido.

Tabla 7. Puntos al final del partido, tiros libres lanzados, tiros libres encestados y porcentaje de aciertos en tiros libres en función del equipo ganador (local o visitante).

\begin{tabular}{|c|c|c|c|c|c|c|}
\hline & & $\mathrm{N}$ & Media & $\begin{array}{l}\text { Desv. } \\
\text { Típica }\end{array}$ & $t$ & $\begin{array}{c}\text { Sig. } \\
p\end{array}$ \\
\hline \multirow{2}{*}{$\begin{array}{l}\text { Tiros } \\
\text { Local }\end{array}$} & Gana local & 38 & 21,21 & 7,95 & \multirow{2}{*}{0,52} & \multirow{2}{*}{, 60} \\
\hline & Gana visitante & 18 & 20,06 & 7,34 & & \\
\hline \multirow{2}{*}{$\begin{array}{l}\text { Tiros } \\
\text { Visitante }\end{array}$} & Gana local & 38 & 18,58 & 6,79 & \multirow{2}{*}{$-0,68$} & \multirow{2}{*}{, 50} \\
\hline & Gana visitante & 18 & 20,11 & 9,95 & & \\
\hline \multirow{2}{*}{$\begin{array}{l}\text { Acierto } \\
\text { local }\end{array}$} & Gana local & 38 & 16,58 & 6,88 & \multirow{2}{*}{0,86} & \multirow{2}{*}{,39 } \\
\hline & Gana visitante & 18 & 15,00 & 5,18 & & \\
\hline \multirow{2}{*}{$\begin{array}{l}\text { Acierto } \\
\text { Visitante }\end{array}$} & Gana local & 38 & 14,00 & 5,60 & \multirow{2}{*}{$-1,12$} & \multirow{2}{*}{, 27} \\
\hline & Gana visitante & 18 & 16,11 & 8,31 & & \\
\hline \multirow{2}{*}{$\begin{array}{l}\text { Porcentaje } \\
\text { Local }\end{array}$} & Gana local & 38 & 76,26 & 8,47 & \multirow{2}{*}{0,20} & \multirow{2}{*}{,84 } \\
\hline & Gana visitante & 18 & 75,78 & 8,99 & & \\
\hline \multirow{2}{*}{$\begin{array}{l}\text { Porcentaje } \\
\text { Visitante }\end{array}$} & Gana local & 38 & 75,29 & 10,53 & \multirow{2}{*}{$-1,50$} & \multirow{2}{*}{,14 } \\
\hline & Gana visitante & 18 & 79,44 & 7,48 & & \\
\hline \multirow{2}{*}{$\begin{array}{l}\text { Puntos finales } \\
\text { Local }\end{array}$} & Gana local & 38 & 82,55 & 9,18 & \multirow{2}{*}{3,44} & \multirow{2}{*}{, 00} \\
\hline & Gana visitante & 18 & 72,50 & 12,16 & & \\
\hline \multirow{2}{*}{$\begin{array}{l}\text { Puntos finales } \\
\text { Visitante }\end{array}$} & Gana local & 38 & 71,05 & 10,14 & \multirow{2}{*}{$-3,11$} & \multirow{2}{*}{,00 } \\
\hline & Gana visitante & 18 & 80,22 & 10,69 & & \\
\hline
\end{tabular}

$p>, 05$ (diferencias no significativas); $\mathrm{p}=, 00$ (diferencias significativas).

\section{Victoria final local o visitante según la relevancia del partido}

En la Tabla 8 se ilustra quién ha sido el equipo ganador en función de la relevancia del partido considerando el cruce y el orden. En los 29 partidos correspondientes al cruce de cuartos, el equipo local resultó vencedor en 18 (62\%) mientras que el visitante lo hizo en 11 partidos (38\%). En los 17 partidos de semifinales, el equipo local resultó vencedor en 15 (88\%) mientras que el visitante lo hizo en 2 partidos (12\%). En los 10 partidos de finales, el equipo local resultó vencedor en $5(50 \%)$ y el visitante lo hizo también en 5 partidos (50\%).

Por lo que respecta al orden de los partidos, en los 30 encuentros correspondientes al primer partido al mejor de tres (o bien $1^{\circ}-2^{\circ}$ al mejor de cinco), el equipo local resultó vencedor en 19 (63\%) mientras que el visitante lo hizo en 11 partidos (37\%). En los 20 partidos correspondientes al segundo encuentro al mejor de tres (o bien $3^{\circ}-4^{\circ}$ al mejor de cinco), el equipo local resultó vencedor en 13 (65\%) mientras que el visitante lo hizo en 7 partidos (35\%). En los 6 partidos correspondientes al tercer encuentro al mejor de tres (o bien $5^{\circ}$ al mejor de cinco), el equipo local resultó vencedor en los $6(100 \%)$. 
Tabla 8. Equipo ganador en función de la relevancia del partido

\begin{tabular}{|c|c|c|c|}
\hline \multicolumn{2}{|c|}{ Relevancia del partido } & \multicolumn{2}{|c|}{$\begin{array}{l}\text { Número de veces como equipo ganador } \\
\qquad(\mathrm{N}=56 \text { partidos })\end{array}$} \\
\hline & & Local & Visitante \\
\hline \multirow{3}{*}{ Cruce } & Cuartos & $18(62,07 \%)$ & $11(37,93 \%)$ \\
\hline & Semifinales & $15(88,2 \%)$ & $2(11,8 \%)$ \\
\hline & Finales & $5(50 \%)$ & $5(50 \%)$ \\
\hline \multicolumn{2}{|l|}{ Total } & $38(67,86 \%)$ & $18(32,14 \%)$ \\
\hline \multirow{3}{*}{ Orden } & $\begin{array}{l}\text { Partido } 1^{\circ} \text { de } 3 \\
\text { ó } 1^{\circ}-2^{\circ} \text { de } 5\end{array}$ & $19(63,3 \%)$ & $11(36,7 \%)$ \\
\hline & $\begin{array}{l}\text { Partido } 2^{\circ} \text { de } 3 \\
\text { ó } 3^{\circ}-4^{\circ} \text { de } 5\end{array}$ & $13(65 \%)$ & $7(35 \%)$ \\
\hline & $\begin{array}{l}\text { Partido } 3^{\circ} \text { de } 3 \\
\text { ó } 5^{\circ} \text { de } 5\end{array}$ & $6(100 \%)$ & $0(0 \%)$ \\
\hline \multicolumn{2}{|l|}{ Total } & $38(67,86 \%)$ & $18(32,14 \%)$ \\
\hline
\end{tabular}

\section{Discusión y conclusiones}

En este estudio nos hemos planteado analizar el número de tiros libres efectuados y el porcentaje de aciertos en función del minuto de juego en que se ejecuta el lanzamiento, de la situación del marcador en el momento de lanzar y del hecho de actuar como equipo local o visitante. También hemos querido comprobar si la relevancia del partido para resolver la eliminatoria guarda relación con el acierto en el tiro libre y con la victoria final.

En relación al minuto de juego en el momento de lanzar, nuestros resultados muestran que en los primeros $5 \mathrm{minu}-$ tos de cada cuarto se lanzan menos tiros libres que en los 5 minutos finales. En cuanto al porcentaje medio de aciertos, no se observan diferencias significativas entre los primeros y segundos tiempos de los partidos. Tampoco hemos obtenido diferencias en el porcentaje de aciertos en función de otras fracciones de tiempo observadas: periodos de cinco minutos, cuartos, tiempos, y entre los 5 primeros minutos del partido y los cinco últimos minutos. Estos datos difieren ligeramente de los aportados Kozar et al. (1995) y Labrador et al. (1995) y coinciden con diferentes estudios que demuestran que el minuto de juego no produce grandes alteraciones en el tiro libre (Montgomery et al., 2008; Ibánez et al., 2009; Uygur et al., 2010).

Hemos observado estabilidad en los porcentajes en todas las franjas temporales, excepto en los cinco primeros minutos del último cuarto de partido. Las razones de esto pueden ser múltiples. Puede que sea el periodo donde se utilicen más a los suplentes, puede que se cargue el juego en mayor medida sobre los jugadores interiores, que poseen un menor porcentaje de acierto en los tiros libres (Gómez y Lorenzo, 2007), tratando de provocar faltas de cara al final de encuentro. En realidad no tenemos datos suficientes para explicar esta tendencia. Tampoco la fatiga podría ser una posible respuesta. No encontramos evidencia de que la fatiga influya en el acierto, coincidiendo con los estudios de Uygur et al. (2010) y Zuzik (2011). Tampoco hemos hallado influencia del tiempo de juego acumulado, incluso tenemos como dato que en los cinco últimos minutos el acierto aumenta, volviendo a las cifras medias de todo el encuentro. La explicación tal vez deba buscarse en cuestiones tácticas que puedan verse reflejadas indirectamente en el tiro libre. En los finales de partido es cuando se realizan más faltas de carácter táctico, y esto se entiende como un factor estresante para un $60 \%$ de los jugadores, siendo para el 100\% estresante el fallar un tiro considerado como fácil (Anshel y Wells, 2000).

En cuanto a la influencia de la situación del marcador en el momento de lanzar en el porcentaje de aciertos, hemos observado una tendencia contraria entre los equipos locales y visitantes. El mayor número de tiros libres lanzados por los equipos locales lo hicieron cuando iban ganando entre 1 y 5 puntos, mientras que los equipos visitantes el mayor número de sus tiros libres cuando iban perdiendo entre 1 y 5 puntos. Además, el mayor porcentaje de veces que los equipos locales lograron el $100 \%$ de aciertos se produjo cuando lanzaron tiros libres con el marcador en contra por más de 10 puntos. Parece que esa amplia desventaja actúa como un elemento que quite presión al jugador local a la hora de encestar. Tal vez pueda pensar que el desenlace del acierto no será decisivo en el marcador. Por su parte, los equipos visitantes lograron más veces el $100 \%$ de aciertos con el marcador en contra entre 1 y 5 puntos. Probablemente ese marcador ajustado active una mayor concentración en el jugador visitante al efectuar 
el lanzamiento. El menor porcentaje de veces que los equipos locales y visitantes lograron el $100 \%$ de aciertos se produjo cuando al lanzar iban perdiendo entre 6 y 10 puntos. El mayor porcentaje de veces que los equipos locales fallaron todos los tiros libres se produjo cuando lanzaron con el marcador empatado lo que pueda deberse a la anticipación de la consecuencia que provoca un incremento en la autoconciencia de la situación, lo que deviene en una disminución del acierto (Whitehead et al., 1996; Dandy et al., 2001; Otten, 2009). En cambio, los equipos visitantes fallaron el mayor porcentaje de veces todos sus tiros libres cuando ganaban por más de 10 puntos. Puede que en este caso esté presente en el jugador el efecto de la relajación por la cómoda situación del marcador.

En resumen, se puede concluir este apartado apuntando que el estado del marcador no ha influido significativamente en el porcentaje de aciertos desde el tiro libre, tanto del equipo local como del equipo visitante, lo que coincide con el estudio de Whitehead et. al (1996).

Se puede apreciar que ir ganando en el marcador entre 6 y 10 puntos es la situación que se asocia con una media más elevada de aciertos desde el tiro libre en los equipos locales mientras que ir ganando de más de 10 es la situación del marcador asociada a una media de aciertos más alta en los equipos visitantes, lo que coincide con las conclusiones de Lafuente (2005). Estos resultados rebaten las conclusiones a las que llegaba Ahart (1973) en su tesis cuando mencionaba que cuando existen diferencias entre 5 y 8 puntos aumenta el porcentaje de acierto, mientras que cuando se queda por encima o por debajo de ese rango, el porcentaje de acierto disminuye. También difieren de lo publicado por otros autores que han afirmado que los porcentajes más altos se dan cuando el marcador está más igualado (Buceta, 1992; Labrador et al., 1995).

Los resultados anteriores parecen indicar que el equipo local con ventajas amplias se relaja, mientras que el visitante en la situación de ventaja se motiva. Esto se produce según diferentes estudios por el grado de concentración o de atención que el deportista es capaz de mantener (Whitehead et al., 1996; Oliveira et al., 2008; Wilson et al., 2009). Cuando se produce una ansiedad cognitiva en forma de preocupación, como ocurre en las situaciones analizadas, aparecen dos efectos principales: por un lado se reduce la capacidad de procesamiento y almacenamiento en la memoria reduciendo los recursos de atención disponible para la tarea, lo que puede provocar un mayor número de errores. Por otro lado la preocupación puede tener un papel de motivación o de activación que estimula el aumento del esfuerzo en la tarea y los recursos y estrategias de procesamiento, pudiendo mejorar el rendimiento (Wilson et al., 2009). Lo anterior nos lleva a concluir que sería necesario para el entrenamiento de los jugadores, conocer el tipo de percepción y respuesta predominante ante estas situaciones, para poder trabajar de manera individualizada en función de las características y demandas del jugador.

En lo referente al hecho de jugar como local o como visitante, nuestros resultados revelan que los equipos locales consiguen al final del partido una media de 79,32 puntos y de ellos 16,07 han sido obtenidos desde el punto de tiros libres (el 20\% aproximadamente). Los equipos visitantes consiguen una media de 74,00 puntos al final del partido, y de ellos 14,68 han sido obtenidos desde el punto de tiros libres (el $19,8 \%$ ). Aunque muy próximos, estos resultados se quedan ligeramente por debajo del $21 \%$ difundido por Cárdenas y Rojas (1997). Además, hemos obtenido que los equipos locales incrementan ligeramente su porcentaje de aciertos en el segundo tiempo con respecto al primero, mientras que los equipos visitantes suelen empeorar ligeramente sus registros en el segundo tiempo. Cuando se ha producido prórroga, los locales suelen bajar el porcentaje de aciertos en ese periodo de prórroga con respecto al segundo tiempo, mientras que los visitantes suelen mejorar sus aciertos en dicho periodo en comparación a sus registros del segundo tiempo. La situación de estrés que puede generar una prórroga en casa, al no solventar el partido en el tiempo reglamentario, parece actuar en contra de los equipos de casa en esta situación contextual concreta cuando los resultados son adversos, tal y como estudiaron Baumeister y Steinhilber (1984), o Tauer, Guenther y Rozek (2009). En consecuencia, entrenar el control de la situación de choking que se produce en esas situaciones de estrés puede tener un efecto significativo sobre el porcentaje de acierto desde el tiro libre, en especial en esos finales igualados donde las diferencias son tan cortas que la anotación puede condicionar el resultado final.

Por lo que respecta a la influencia de la relevancia del partido (cruce y orden del partido en la eliminatoria) sobre el porcentaje de acierto en el tiro libre y sobre la victoria final, nuestros datos revelan que el cruce de semifinales es el contexto donde el equipo local ha obtenido el mayor porcentaje de victorias. Por el contrario, en la eliminatoria de la final se ha producido un mayor equilibrio en el número de victorias locales o visitantes. Parece como si el equipo local estuviera más presionado cuando está muy próximo el desenlace de conseguir o perder un título pero no es el último partido de la eliminatoria, datos que coinciden con los obtenidos por Lafuente (2005). También se puede deber a un aumento de la motivación del equipo visitante como afirma el estudio de Štrumbelj et al. (2011). En este último partido el equipo local sí suele conseguir la victoria. Un dato llamativo es que, tanto el equipo local como el visitante, suelen ganar si anotan en torno a 16 o más puntos desde el tiro libre y suelen perder si anotan menos de 16 puntos desde el tiro libre. Las diferencias encontradas entre los equipos locales y los visitantes, en relación a los tiros libres intentados, no pueden considerarse importantes. En cuanto a los porcentajes de aciertos, existe 
una mínima diferencia favorable a los equipos que juegan en cancha propia. Todos estos datos coinciden los obtenidos por Cárdenas y Rojas (1997).

Podemos afirmar que el tiro libre constituye, dentro del marco competitivo, una de las situaciones más importantes de juego en baloncesto. Los resultados antes expuestos están condicionados por el tipo de muestra. En este sentido, es posible que realizando el mismo análisis aplicado a otros niveles de competición encontráramos diferencias considerables. Desde esta perspectiva, la media de los porcentajes de aciertos entre los equipos ganadores y perdedores en las denominadas categorías inferiores, posiblemente muestren diferencias algo más llamativas, y por tanto, las conclusiones serían distintas.

A pesar de ello, entendemos que los datos obtenidos son significativos del máximo nivel de competición que tenemos en nuestro país y como consecuencia, son orientativos tanto para los que trabajan en este nivel como para los que desarrollan su labor profesional en la formación de jóvenes jugadores.

Consideramos que el tiro libre debe ser entrenado en gran medida dentro del volumen total de entrenamiento. Para poder mejorar la cantidad de puntos obtenidos mediante tiros libres, en primer lugar, el equipo debe ser capaz de generar aquellas situaciones que obliguen a los contrarios a cometer faltas, y además los jugadores deberán mejorar sus porcentajes de eficacia. Además, debemos estudiar en profundidad las causas que impiden el desarrollo de las capacidades técnicas y psicológicas implicadas en el tiro libre.

Tal como aportan algunos estudios (Kozar et al.,1995; Cárdenas y Oña, 1996) los métodos de entrenamiento utilizados habitualmente no permiten el desarrollo de las variables psicológicas implicadas en este tipo de lanzamiento. Rodríguez y Sáez (2009) concluyen que es la concentración la que principalmente incide en la mayor o menor eficacia en el tiro libre en situación de competición, y esta concentración no es la misma en las situaciones de entrenamiento.

Desde la Psicología del Deporte se han desarrollado una serie de técnicas y pautas que constituyen para entrenadores y jugadores una valiosa ayuda para el entrenamiento y la competición en baloncesto. Así por ejemplo, Rivarés (1996) ha desarrollado un entrenamiento de tiro libre basado en la mejora de la concentración en el mismo. Es un entrenamiento de utilidad para jugadores que ya tienen una mecánica de tiro adquirida y se basa en la importancia atribuida a la concentración en el momento de efectuar el tiro.

Está comprobado que no existen estrategias o técnicas de concentración en el tiro libre que sean óptimas para todos los jugadores, por el contrario, cada jugador tiene o puede tener una estrategia de concentración que le conduzca a optimizar su efectividad (Fierro, 2003). Sería necesario para el entrenamiento de los jugadores, conocer el tipo de percepción y respuesta predominante ante las situaciones de ansiedad cognitiva, para poder trabajar de manera individualizada en función de las características y demandas del jugador y así aplicar mejores estrategias del entrenamiento del tiro libre.

La mejora y puesta a punto de la condición psicológica pasa por el conocimiento de qué variables son las fundamentales en cada momento y de qué manera puede el entrenador manejarlas y entrenarlas. El conocimiento de las estadísticas de juego permite conocer los aspectos que deben ser más entrenados. En este sentido, consideramos de gran interés una revisión profunda de la metodología actual de entrenamiento del tiro libre con el objetivo de recopilar programas de intervención encaminados a la mejora de la eficacia de este tipo de lanzamiento.

\section{Referencias}

1. Ahart, F. C. (1973). The effect of score differential on basketball free throw shooting. Ithaca College: Master's.

2. Anshel, M. H. y Wells, B. (2000). Sources of acute stress and coping styles in competitive sport. Anxiety, Stress and Coping, 13, 1-26.

3. Arias, J. L., Argudo, F. M., Alonso, J. I. (2012). Effect of basketball mass on shot performance among 9-11 year-old male players. International Journal of Sports Science \& Coaching. 7(1), 69-79.

4. Balmer, N. J., Nevill, A. y Williams, M. (2001). Home advantage in the Winter Olympics (1908-1998). Journal of Sports Sciences, 19, 129-139.

5. Baumeister, R. F. y Steinhilber, A. (1984). Paradoxical effects of supportive audiences on performance under pressure: The home field disadvantage in sports championships. Journal of Personality and Social Psychology. 47, 85-93.

6. Bray, S. R. y Widmeyer, W. N. (2000). Athlete's perceptions of the home advantage: An investigation of perceived causal factors. Journal of Sport Behavior, 23 (1), 1-10.

7. Buceta, J.M. (1992). Intervención psicológica con el equipo nacional olímpico de baloncesto femenino. Revista de Psicología del Deporte, 2, 70-87.

8. Cárdenas, D. y Ońa, A. (1996). Las estrategias de práctica del tiro libre basadas en la utilización del refuerzo positivo. Congreso Andaluz de Psi- cología del Deporte. Facultad de Ciencias de la Educación. Departamento de Psicología. Cádiz.

9. Cárdenas, D. y. Rojas, J. (1997). Determinación de la incidencia del tiro libre en el resultado final a través del análisis estadístico. Revista Motricidad, 3, 177-186.

10. Cardenas, D. (1998). El entrenamiento del tiro libre. Revista RED Tomo XI, n 4, 29-35.

11. Courneya, K. S. y Carron, A. V. (1992). The home advantage in sports competitions: A literatura review. Journal of Sport \& Exercise Psychology, 14, 13-27.

12. Dandy, J., Brewer, N. y Tottman, R. (2001). Self-conciousness and perfomance decrements within a sporting context. The Journal of Social Psychology. 141(1), 150-152.

13. Fierro, C. (2002). Variables relacionadas con el éxito deportivo en las ligas NBA y ACB de baloncesto. Revista de Psicología del Deporte, 11(2), 247-255.

14. Fierro, C. (2003). Técnicas de intervención psicológica en baloncesto. En A. Hernández (Coord.), Psicología del Deporte (Vol. III), Aplicaciones 1, pp. 103-117. Recuperado de http://www.efdeportes.com.

15. Foster, D. J. y Weigand, D. A. (2006). The effect of removing superstitious behaviour and introducing a pre-perfomance routine on bas- 
ketball free throw perfomance. Journal of Applied Sport Psychology, 18, $167-171$.

16. Getz, G.E. y Rainey, D.W. (2001). Flexible short-term goals and basketball shooting performance. Journal of Sport Behaviour, 24(1), 31-41.

17. Gómez, M. A. y Lorenzo, A. (2007). Análisis discriminante de las estadísticas de juego entre bases, aleros y pívots en baloncesto masculino. Apunts, 87, 86-92.

18. Gómez, M. A.; Pollard, R., (2011). Reduced home advantage for basketball teams from capital cities in Europe. European Journal of Sport Science. Vol. 11 (2). 143-148.

19. Gooding, A. y Gardner, F. L. (2009). An investigation of the relationship between mindfulness preshot routine and basketball free throw percentage. Journal of Clinical Sport Psychology, 4, 303-319.

20. Harle, S. K. y Vickers, J. N. (2001). Training quiete ye improves acuraccy in the basketball free throw. The Sport Psychologist, 15, 289-305.

21. Hays, D. y Krause, J. V. (1987). Score on the throw. The Basketball Bulletin, Winter, 4-9.

22. Ibánez, S. J., Sampaio, J., Feu, S., Lorenzo, A., Gómez, M. A. y Ortega, E. (2008). Basketball game related statistics that discriminate between team's season-long succes. European Journal of Sport Scienc, 8(6), 369372.

23. Ibánez, S. J., García, J., Feu, S., Parejo, I. y Cañadas, M. (2009). La eficacia del tiro a canasta en la NBA: análisis multifactorial. Revista Cultura Ciencia y Deporte, 4, 39-47.

24. Ittenbach, F. y Esters, I. G. (1995). Utility of team indices for predicting end of season ranking in two national polls. Journal of Sport Behaviour, 18(3), 216-224.

25. Jenkins, R. (1977). Win the big ones from the foul line. Scholastic Coach, 47(5), 42, 88-89.

26. Jones, S. (2007). Drills to improve free throw percentage. FIBA Assist Magazine, 28, 4-10.

27. Keetch, K. M., Lee, T. D. y Schmidt, R.A. (2008). Especial Skills: Specifity embedded within generality. Journal of Sport and Exercise Psycho$\log y, 30,723-736$.

28. Kozar, B., Vaughn, R. E., Whitfield, K. E., Lord, R. H. y Dye, B. (1994). Importance of free-throws at various stages of basketball games. Perceptual and Motor Skills, 78(1), 243-248.

29. Kozar, B., Vaughn, R. E, Lord, R. H, y Whitfield, K. E,. (1995). Basketball free-throw performance: practice implications. Journal of Sport Behavior, 18(2), 123-129.

30. Krendl, A., Gainsburg, I., Ambady, N. (2012). The effect of stereotypes and observer pressure on athletics perfomance. Journal of Sport \& Exercise Psychology. 34, 3-15.

31. Labrador, F. J., Crespo, M., Buceta, J. M. y González, S. (1995). Factores contextuales implicados en el lanzamiento de tiros libres en baloncesto. Revista de Psicología del Deporte, 8, 87-99.

32. Lafuente, V. (2005). Factores contextuales y personales en el rendimiento en los tiros libres en baloncesto. Tesis doctoral no publicada. UNED. Madrid.

33. Larumbe, E. (2001). Entrenamiento de variables psicológicas para los tiros libres. Revista de Psicología del Deporte, 10(1), 89-97.

34. Lonsdale, C. y Tam, J. T. (2008). On the temporal and behavioural consistency of pre-perfomance routines: an intra-individual analisys of elite basketball player's free throw shooting accuracy. Journal of Sport Sciences, 26(3), 259-266.

35. Lorenzo, A., Gómez, M. A., Ortega, E., Ibánez, S. y Sampaio, J. (2010). Game related statistics which discriminate between winning and losing under-16 male basketball games. Journal os Sport Science and Medicine, 9, 664-668.

36. Lorenzo, A., Gómez, M. A., Sampaio, J. (2003). Análisis descriptive de las posesiones de 24 segundos en baloncesto. Revista Digital efdeportes. Año 9, 67. Recuperado de: http://www.efdeportes.com/efd67/balonc. htm
37. Mesagno, C., Marchant, D. y Morris, T. (2009). Alleviating choking: the sounds of distraction. Journal os Applied Sport psychology, 21, 131147.

38. Montgomery, P., Pyne, D., Hopkins, W., Dorman, J., Cook, K. y Minahan, C. (2008). The effect of recovery strategies on physical perfomance and acumulative fatigue in competitive basketball. Journal of Sport Science, 26(11), 1135-1145.

39. Montero, I. y León, O. G. (2002). Clasificación y descripción de las metodologías de investigación en psicología. International Journal of Clinical and Health Psychology, 2, 503-508.

40. Moore, J. C. y Brylinsky, J. (1995). Facility familiarity and the home advantage. Journal of Sport Behavior, 18 (3), 302-311.

41. Navarro, R. M., Lorenzo, A., Gómez, M. A. y Sampaio, J. (2009). Analysis of critical moments in the league ACB 2007-2008. Revista de Psicologia del Deporte, 18(3), 391-395.

42. Okubo, H. y Hubband, M. (2006). Dynamics of the basketball shot with application to the free throw. Journal of Sport Science, 24(12), 1303-1314.

43. Oliveira, R. F., Oudejans, R. D. y Beek, P. J. (2008). Gaze behaviour in basketball shooting: further evidence for online visual control. Research Quarterly for Exercise \& Sport, 79(3), 399-408.

44. Ortega, E., Cárdenas, D., Puigcerver, C. y Méndez, J. J. (2005). Propuestas prácticas para el entrenamiento del tiro libre en etapas de formación elaboradas a partir del análisis de la competición. Cuadernos de Psicología del Deporte, 5(1), 197-217.

45. Otten, M. (2009). Choking vs clutch perfomance: A study of sport perfomance under pressure. Journal os Sport and Exercise Psychology, 31, 583-601.

46. Pickens, M. (1994). Game location as a degterminant of team performance in ACC basketball during 1990-1991. Journal of Sport Behavior, $17(4), 212-216$

47. Rivares, L (1996). La concentración en el tiro libre. Revista de Psicología del Deporte, 9, 77-90.

48. Sampaio, J., Drinkwater, E. J. y Leite, N. M. (2010). Effects of season period, team quality, and playing time on basketball players' gamerelated statistics. European Journal of Sport Science, 10(2), 141-149.

49. Sampaio, J., Fraga, F., Silva, T. (2004). La evolución de las estadísticas de los tiros libres en partidos de baloncesto de formación. III Congreso de la Asociación Española de Ciencias del Deporte. Valencia, 11-13 de marzo.

50. Sampaio, J. y Janeira, M. (2003). Importance of free throw perfomance in game outcome during the final series of basketball playoffs. International Journal of Applied Sport Sciences, 15(2), 9-16.

51. Sánchez, A., González, E., Ruiz, M., San-Juan, M., Abando, J., De Nicolás, L. y García, F. (2001). Estados de ánimo y rendimiento deportivo en fútbol: jexiste la ventaja de jugar en casa?. Revista de Psicología del Deporte, 10 (2), 197-209.

52. Schneider, R.C., Williams, C. (2010). Success rates of underhand and overhand free-throws as novel skills. Journal of Physical Education and Sport. 27(2), 13-20.

53. Silva, J. M. y Andrew, J. A. (1987). An analysis of game location and basketball performance in the atlantic coast conference. International Journal of Sport Psychology, 18, 188-204.

54. Smith, R. (2005). Disconnects between popular discourse and home advantage research: What can fans and media tell us about the home advantage phenomenon? Journal of Sports Sciences, 23 (4), 351-364.

55. Štrumbelj, E., Vračar, P., Robnik-Šikonja, M., Dežman, B., Erčulj, F. (2011). Statistična analiza prednosti domačega igrišča in vplivov spremembe pravil v 1 . A in 1. B slovenski moški košarkarski ligi. Revija $Z a$ Teoreticna in Prakticna Vprasanja Sporta, 59 Issue 3/4, p102-106.

56. Tauer, J. M., Guenther, C. L. y Rozek, C. (2009). Is there a home choke in decisive playoff basketball games? Journal of Applied Sport Psychology, 21, 148-162. 
57. Tran, C. M. y Silverberg, L. M. (2008). Optimal reléase condictions for the free throw in men's basketball. Journal os Sports Sciences, 26(11), $1147-1155$.

58. Uygur, M., Gottepe, A., Ak, E., Karabörk, H. y Korkusuz, F. (2010). The effect of fatigue on the kinematics of free throw shooting in basketball. Journal of Human Kinetics, 24, 51-56.

59. Varca, P. E. (1980). An analysis of home and away game performance of male college basketball teams. Journal of Sport Psychology, 2 (3), 245-257.

60. Walker, F. (1985). Take P.R.I.D.E. in your free-throwing: It will win for you. Scholastic Coach, 55(3), 18-20.

61. Whitehead, R., Butz, J. W., Kozar, B. y Vaughn, R. E. (1996). Stress and perfomance: An application of Gray's three-factor arousal theory to basketball free throw shooting. Journal os Sport Science, 14, 393-401.

62. Wilson, M. R., Vine, S. J. y Wood, G (2009). The influence of anxiety o visual attentional control in basketball free throw. Journal of Sport Exercise Psychology, 31, 152-168.

63. Zuzik, P. (2011). Free throw shooting effectiveness in basketball matches of men and women. Sport Science Review. 20(3-4), 149-160.

\section{Apéndice}

Relación de encuentros analizados. Muestra de Partidos de Play-Offs

\begin{tabular}{|c|c|c|}
\hline \multicolumn{3}{|c|}{ TEMPORADA } \\
\hline $2007-2008$ & 2008-2009 & $2009-2010$ \\
\hline Joventut-Girona (Cuartos) & Barcelona-Pamesa (Cuartos) & Cajalaboral-Estudiantes (Cuartos) \\
\hline Girona-Joventut (Cuartos) & Pamesa-Barcelona (Cuartos) & Estuiantes-Cajalaboral (Cuartos) \\
\hline Joventut-Girona (Cuartos) & Tau-Bilbao (Cuartos) & R. Madrid-Cajasol (Cuartos) \\
\hline Barcelona-Bilbao (Cuartos) & Bilbao-Tau (Cuartos) & Cajasol-R. Madrid (Cuartos) \\
\hline Bilbao-Barcelona (Cuartos) & Unicaja-Gran Canaria (Cuartos) & R. Madrid-Cajasol (Cuartos) \\
\hline Tau-Pamesa (Cuartos) & Gran Canaria-Unicaja (Cuartos) & Barcelona-Gran Canaria (Cuartos) \\
\hline Pamesa-Tau (Cuartos) & Unicaja-Gran Canaria (Cuartos) & Gran Canaria-Barcelona (Cuartos) \\
\hline Tau-Pamesa (Cuartos) & R. Madrid-Joventut (Cuartos) & Valencia-Unicaja (Cuartos) \\
\hline R. Madrid-Unicaja (Cuartos) & Joventut-R. Madrid (Cuartos) & Unicaja-Valencia (Cuartos) \\
\hline Unicaja-R. Madrid (Cuartos) & R. Madrid-Joventut (Cuartos) & Barcelona-Unicaja (Semifinal) \\
\hline Dkv Joventut-Barcelona (Semifinal) & Barcelona-Unicaja (Semifinal) & Barcelona-Unicaja (Semifinal) \\
\hline Barcelona-Dkv Joventut (Semifinal) & Unicaja-Barcelona (Semifinal) & Cajalaboral-R. Madrid (Semifinal) \\
\hline Tau Cerámica-Unicaja (Semifinal) & Barcelona-Unicaja (Semifinal) & Cajalaboral-R. Madrid (Semifinal) \\
\hline Unicaja-Tau Cerámica (Semifinal) & Tau-R. Madrid (Semifinal) & R. Madrid-Cajalaboral (Semifinal) \\
\hline Barcelona-Tau (Final) & R. Madrid-Tau (Semifinal) & R. Madrid-Cajalaboral (Semifinal) \\
\hline Barcelona-Tau (Final) & Tau-R. Madrid (Semifinal) & Cajalaboral-R. Madrid (Semifinal) \\
\hline \multirow[t]{4}{*}{ Tau-Barcelona (Final) } & Tau-Barcelona (Final) & Barcelona-Cajalaboral (Final) \\
\hline & Tau-Barcelona (Final) & Barcelona-Cajalaboral (Final) \\
\hline & Barcelona-Tau (Final) & Cajalaboral-Barcelona (Final) \\
\hline & Barcelona-Tau (Final) & \\
\hline
\end{tabular}

